\title{
Synthetic and Systems Biology Approach towards Designing Metabolic Bypass and Identifying Novel Enzymes for Cholesterol Lowering Drug Precursor (BTO) Biosynthesis from Crude Glycerol
}

\section{Dipankar Ghosh}

Department of Life Sciences, Sir Alexander Fleming Building, Imperial College London SW7 2AZ, United Kingdom.

\begin{tabular}{|c|c|}
\hline ARTICLE INFO & ABSTRACT \\
\hline $\begin{array}{l}\text { Article history: } \\
\text { Received on: } 19 / 06 / 2017 \\
\text { Accepted on: } 15 / 09 / 2017 \\
\text { Available online: } 30 / 10 / 2017\end{array}$ & $\begin{array}{l}\text { 1, 2, 4-Butanetriol (BTO) is a potential precursor of Cholesterol Lowering Drugs. BTO production is } \\
\text { predominantly dependent on chemical conversions at present. Biological route for BTO biosynthesis suffers due } \\
\text { to multiple issues like unavailability of efficient biosynthetic pathways, inefficient enzymes, usage of expensive } \\
\text { edible feedstock and unavailability of suitable chassis host strains. These issues practically restrict its large scale }\end{array}$ \\
\hline $\begin{array}{l}\text { Key words: } \\
\text { 1, 2, 4-Butanetriol, } \\
\text { Cholesterol Lowering Drug, } \\
\text { Biodiesel Waste, Crude } \\
\text { Glycerol, Chassis Strain, } \\
\text { Biosynthetic Pathway. }\end{array}$ & $\begin{array}{l}\text { The main aim of this study is to find out efficient novel biosynthetic pathways which can convert non-edible } \\
\text { feedstock (crude glycerol from biodiesel waste) towards BTO biosynthesis. The second aim of this study is to } \\
\text { identify putative non-natural enzyme classes predicted to be involved in these biosynthetic pathways. Future } \\
\text { perspective will be to functionalize these pathways towards In-vivo validation within suitable metabolically } \\
\text { engineered microorganisms for improving BTO biosynthesis. }\end{array}$ \\
\hline
\end{tabular}

\section{INTRODUCTION}

1, 2, 4-Butanetriol (BTO) is a potential precursor of Cholesterol Lowering Drug (CLD) i.e. Crestor and Zetia. Effective target for CLDs is HMG-CoA reductase enzyme to block cholesterol biosynthesis in liver. Biomolecular mode of action of CLDs has already been established (Fig 1). BTO is not only used as a precursor of CLDs but also in synthesis of energetic plasticizer material $(\mathrm{D}, \mathrm{L}$-butanetriol trinitrate as a replacement for nitroglycerin), cationic lipids (towards drug delivery), polyurethane foams as natural fiber (having improved elastic properties and similar compression-bending characteristics), 3-hydroxytetrahydrofuran or dehydrated form of BTO (key component for the HIV drug Amprenavir) as well

\footnotetext{
* Corresponding Author

Dipankar Ghosh, Department of Life Sciences, Sir Alexander Fleming Building, Imperial College London SW7 2AZ, United Kingdom, Tel Office: +44-2075945213. Email:d.ghosh@imperial.ac.uk
}

(Ren and Liu, 1999; Lau, 2007; Mandava et al., 2012; Yamaguchi et al., 2008; Gouranlou and Kohsary, 2010). In chemical point of view, BTO is an alcohol having three hydrophilic alcoholic hydroxyl groups and chiral property, with two possible enantiomers. BTO has been mass-produced following few traditional chemical routes utilizing glycidol; 2-butene-1, 4-diol; 3, 4-dihydroxybutanoate or Malate; D-glucose as the initial chemical substrates (Adkins and Billica, 1948; Campbell, 1983; Ikai et al., 2005; Kwak et al., 2005; Molefe, 2005; Monteith et al., 1998; Ritter, 2004). Stoichiometric reduction of esterified malic acid (diethyl D, L-malate) using Sodium borohydride $\left(\mathrm{NaBH}_{4}\right)$ is a widely used industrial method for BTO production (Ikai et al., 1999).

However, this process requires $\mathrm{NaBH}_{4}$ as the reducing agent. For each ton of BTO to be synthesized, multiple tons of borate salts are generated as the by-products, thus resulting in high production costs and severe environmental pollution (Frost and Niu, 2011). 


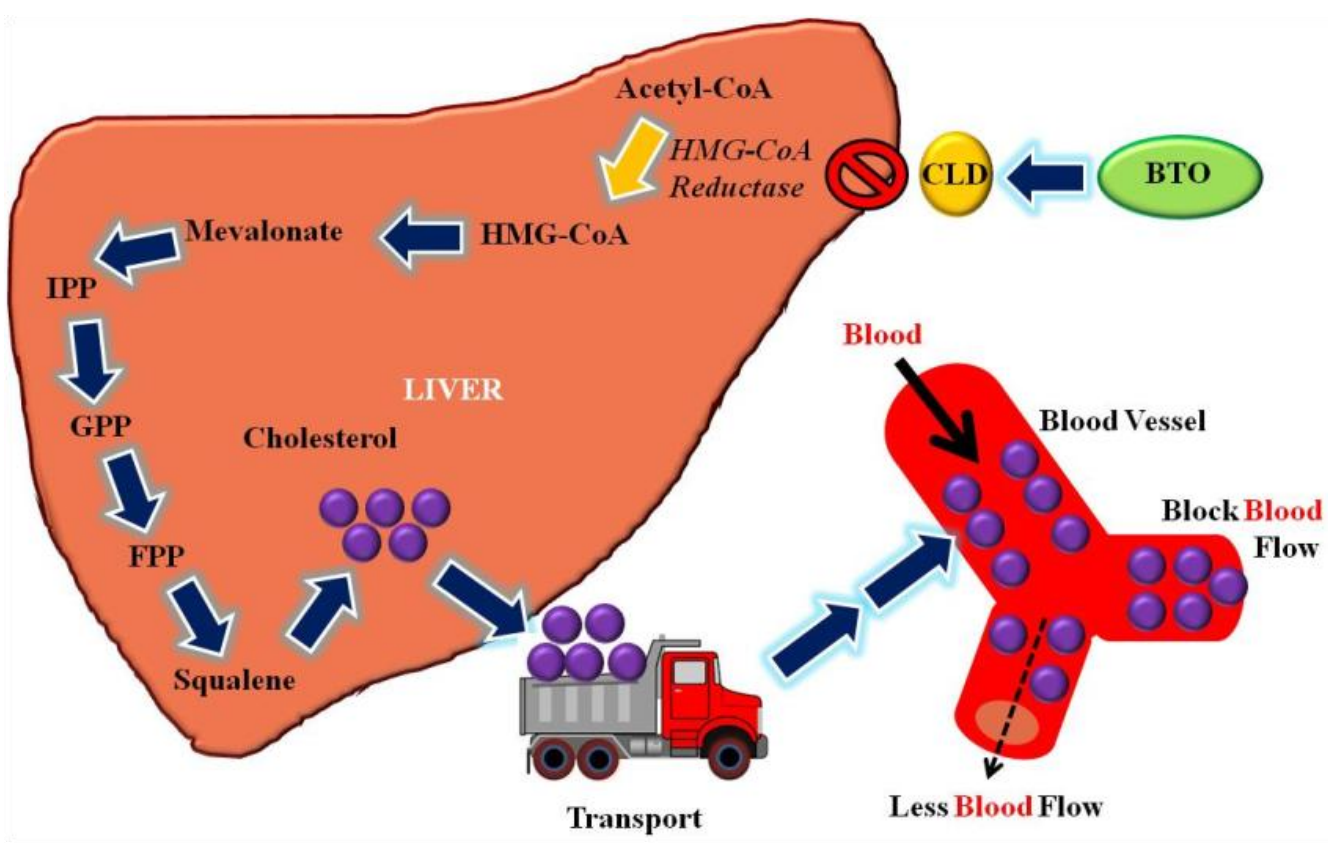

Fig 1: Biological mode of action of BTO as a cholesterol lowering drug.

Catalytic hydrogenation of malate over rubidium and carbon is another alternate; though hydrogenation reactions have some physiological constraints like 2900-5000 psi pressure, elevated temperature that results into undesirable toxic byproducts (Niu et al., 2003). Moreover, all of these traditional chemical procedures of BTO synthesis suffer from several bottlenecks i.e. extreme reaction conditions, multiple steps, and poor selectivity (Antons et al., 2002). These critical problems associated with traditional industrial synthesis actually hinder further applications of petrochemical-based BTO generation.

Therefore, Biological route for BTO biosynthesis can be a green alternative. There are several promising microbial catalysts in nature that synthesize optically active BTO like S-isomer producers (Bacillus subtilis, Candida succiphila, Comamonas acidovorans, Cryptococcus curiosus, Cryptococcus humicolus, Cryptococcus neoformans, Dekkera custersianus, Eremascus fertilis, Hansenula glucozyma, Hansenula polymorpha, Schizoblastosporion kobayasii, Stephanoascus ciferrii, Sterigmatomyces elviae, Sterigmatomyces polyborus) and Risomer producers (Geotricum candidum, Geotricum fermentans, Sterigmatomyces halophiles, Trichosporon cutaneum, Yarrowia lipolytica) (Yamada-Onodera et al., 2007). The major starting materials for BTO biosynthesis are ligocellulosic biomass, C5 sugars (D-xylose, L-arabinose), and amino acid (malate, a microbial tricarboxylic acid cycle intermediate). The most well established biological de novo or chimerical metabolic pathways are reconstituted and functionalized in Escherichia coli, Arabidopsis thaliana (Fig 2).

The first microbial biosynthesis approach has shown that $6.2 \mathrm{~g} / \mathrm{L}$ of D-BTO produces from $20 \mathrm{~g} / \mathrm{L}$ D-xylose which is $30 \%$ molar yield; $8.3 \mathrm{~g} / \mathrm{L}$ of D-BTO from $28 \mathrm{~g}$ of D-xylonic acid or
$45 \%$ molar yield and L-arabinonic acid (10 g/L) into L-BTO (2.4 g/L) resulting into 35\% yield (Niu et al., 2003; Frost and Niu, 2008). Afterwards several microbial engineering techniques have been applied but that did not overcome existing production yield (Valdehuesa et al., 2014; Cao et al., 2015; Zhang et al., 2016). Various chaperone proteins (DnaK-DnaJ-GrpE; GroES-GroEL; Trigger factor) have also been co-expressed in pre-established BTO biosynthesizing E.coli chassis, but there is no improvement obtained (Lu et al., 2016). Moreover, these well established synthetic metabolic pathways were implemented in higher plant Arabidopsis thaliana resulting into low production with $20 \mathrm{mg}$ of BTO per gram of soil-grown plants (Abdel-Ghany et al., 2013). A synthetic bacterial metabolic network has also been reconstituted for improved synthesis of BTO from malate, a Tricarboxylic acid cycle (TCA) cycle metabolic intermediate (In this case, the production was negligible i.e. $180 \mathrm{ng} / \mathrm{L}$ ) (Li et al., 2014).

In this current scenario, an alternative feedstock "Glycerol" may be tried out towards improving BTO productivity and molar yield. Thus far a very vital question arises "why should we think for an alternative potential feedstock glycerol rather than Xylose or Arabinose?" The first and foremost reason is that different microbial regimes can uptake glycerol as carbon and energy source; mediated by the glycerol diffusion facilitator, an integral membrane protein catalyzing the rapid equilibration of concentration gradients of glycerol across the cytoplasmic membrane under specified physiological growth conditions towards high value added biomolecules generations (Beijer et al., 1993). These potential glycerol utilizing microbes are Klebsiella pneumoniae, Clostridium butyricum, Lactobacillus leuteri, Pseudomonas sp., Cupriavidus sp, Escherichia coli, Saccharomyces cerevisiae, Schizosaccharomyces pombe, 
Rhodopseudomonas palustris, Chlorella vulgaris, Synechocystis sp. PCC 6803, Synechococcus sp. PCC 7002, Spirulina platensis, Chlamydomonas reinhardtii and Nannochloropsis sp. (Casella et al., 2016; Dharmadi et al., 2006; Ghosh et al., 2012a; Ghosh et al., 2012b; Kong et al., 2013; Lambert and Stevens, 1986; May et al., 1982; Murarka et al, 2008; Narayan et al., 2005; Swinnen et al., 2013; Taghavi et al., 2016). These glycerol utilizing microbes could be the potential hosts for chassis strain development and for functionalization of novel hypothetical biosynthetic pathways towards improving BTO productions. Biodiesel manufacturing generates large quantity of crude glycerol $(10 \mathrm{~kg} / 100 \mathrm{~L})$ as a major by-product, which has great impact on environmental pollution concerning disposal issues. First generation biofuel i.e. biodiesel production increases each year with a world total production of about 15 billion liters in 2009. A projection has shown that world biodiesel market possibly would reach 37 billion gallons by 2016 , which implies generation of 4 billion gallons of crude glycerol as predominant side product (Kerr et al., 2007). However, crude glycerol price decreases from 0.25 dollar to 0.05 dollar per pound (Thompson and He, 2006; Johnson and Taconi, 2007; Yang et al., 2012). Hence, this current global scenario makes crude glycerol as a potentially relevant and cheap green non-edible feedstock for developing sustainable bioprocess towards high value added biomolecular production.

The major objective of this current study is to design or predict novel hypothetical biosynthetic bypass pathway utilizing glycerol as major feedstock. Moreover, study will be extended towards identification of class of novel enzymes.

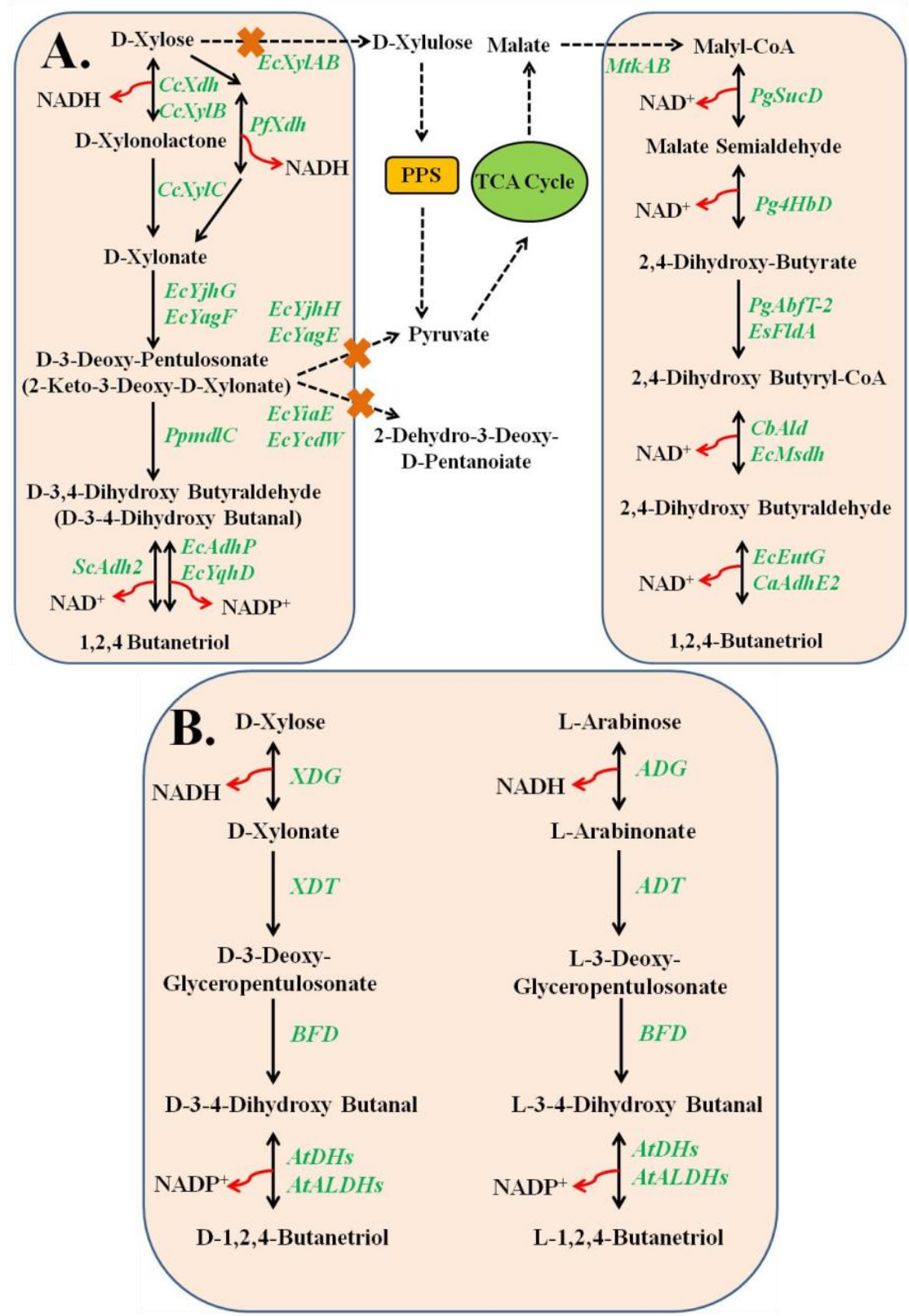

Fig 2: BTO biosynthesis from D-xylose, L-arabinose and malate as feedstock (A) in Escherichia coli and (B) in Arabidopsis thaliana. 


\section{MATERIALS AND METHODS}

\section{Metabolic pathway prediction tools and databases}

In this current study, biosynthetic metabolic networks have been designed using Kyoto Encyclopedia of Genes and Genomes (KEGG) pathway map, From Metabolite to Metabolite (FMM) \& Metabolic Route Explorer (MRE) pathway finders and eEquilibrator system biology tools. KEGG pathway map represents a high level function of networks of molecular interactions, enzymatic reactions and relations in terms of graphical format. KEGG pathway map tool helps to draw reference pathway maps together with organism specific pathway maps that are computationally generated by matching KEGG Orthology (KO) assignments in the genome with reference pathways.

KOs refers to sequence similarity groups as well as functional orthologs. Three major segments i.e. metabolic pathways, gene clusters, and phylogeny have always been considered while defining KOs (Kanehisa et al., 2017). FMM reconstructs metabolic pathways form one metabolite to another metabolite among diverse groups of species depending on the KEGG database and other integrated biological databases (UniprotKB, Swissprot, dbPTM etc) (Chou et al., 2009). MRE is another system biology tool used in this study to suggest foreign enzymes for the biosynthesis pathway design with competing endogenous reactions.

It is an effective tool to guide the design and optimization of heterologous biosynthesis pathways providing pathway ranking scores and competing naive reactions. MRE workflow functions considering KEGG databases for biochemical transformations and Boltzmann factor for thermodynamic considerations (Kuwahara et al., 2016) within the web based interface.

\section{Thermodynamic constraint prediction tool}

eQuilibrator is a biochemical thermodynamics calculator for individual enzymatic steps involved within the large metabolic networks. eQuilibrator is effective online system biology search tool which connects a comprehensive and accurate database of thermodynamic properties of biochemical compounds and enzymatic reactions. It empowers easy Gibb's energies (in $\mathrm{Kcal} / \mathrm{mol}$ units) calculation of compounds and reactions considering arbitrary $\mathrm{pH}$, ionic strength and metabolite concentrations in an online interactive interface (Flamholz et al., 2012).

\section{RESULTS AND DISCUSSION}

All in-silico results have been generated and designed based on a simple workflow (Fig 3). The workflow consists of query input, auto execution (using different system biology web tools), and probable outputs considering thermodynamic constraints and enzymatic reaction network logistics.

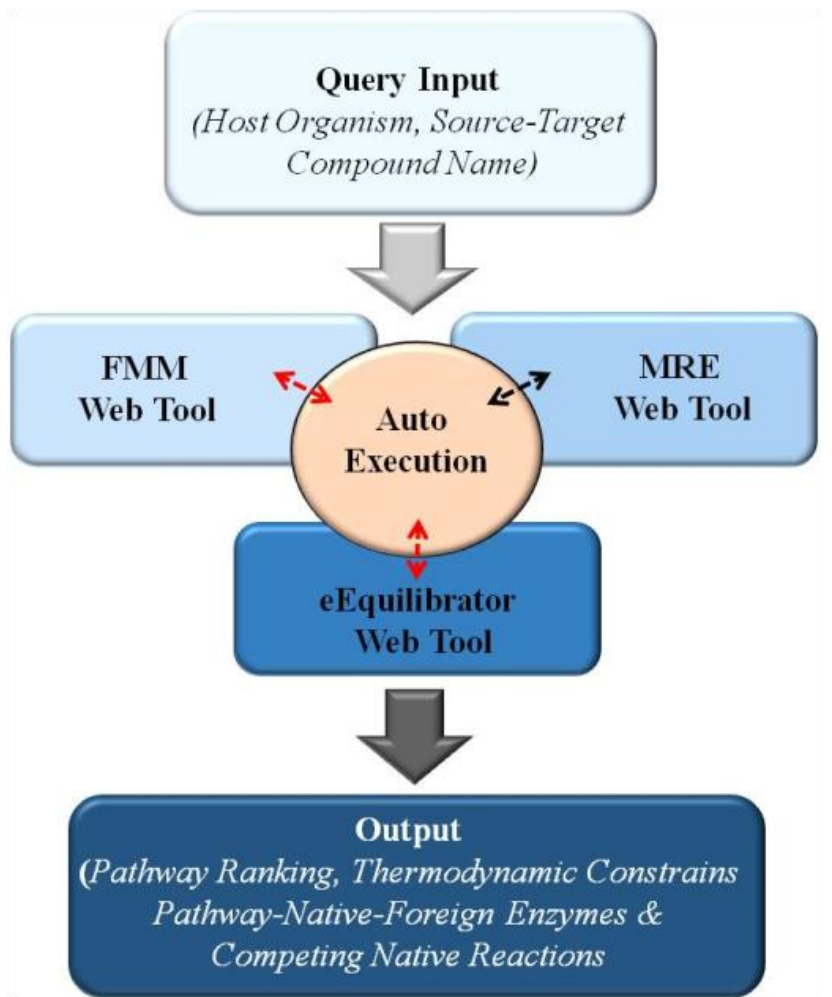

Fig 3: System biology (web based) workflow for prediction and design of biosynthetic metabolic pathways.

\section{Glycerol entry point node}

In this current study, glycerol to BTO biosynthetic pathways are predicted and enzymatic networks has been designed respectively through two major routes of glycerol entry points likely Methylglyoxal and Lactaldehyde nodes (Fig 4). As far thermodynamic constraints it seems Methylglyoxal and Lactaldehyde nodes are quiet energetically favorable (concerning Gibb's free energy change). Each class of enzyme in these two nodes represents different sub-classes of enzymes from diverse microbial regimes including human and plants (enzyme dataset can be obtained following web servers). In addition, a competing D-fuconate node has also been identified which redirects the flux of Pyruvate and Lactaldehyde towards D-fuconate through 2dehydro-3deoxy-L fuconate intermediate formation. However, Dfuconate conversion from 2-dehydro-3deoxy-L fuconate is highly energetically unfavorable step. Even though predicted design of this synthetic pathway looks promising but still few reaction steps need to be further re-evaluated as those reactions are energetically unfavorable (i.e. Pyruvate to D-Xylonate and Lactaldehyde to Methylglyoxal reaction steps). The most possible solution space for this issue (having energetically unfavorable enzymatic steps) is to introduce energy coupled enzymatic reaction (i.e. Adenosine triphosphate or ATP hydrolysis along with carboxylation and/or redox reactions) which may turn the overall reaction energetically favorable (Atkinson, 1977). Furthermore, two putative balanced equations (i.e. reaction 1: 2-dehydro-3deoxy- $\mathrm{L}$ fuconate to 2 dehydro-3deoxy-D-xylonate and reaction 2: D-fuconate to $\mathrm{D}$ Xylonate) have manually been derived using eEqullibriator tool. It 
shows that both reactions (reaction $1 \& 2$ ) are highly energetically unfavorable redox reactions having Gibb's free energy change values $35.4 \mathrm{kcal} / \mathrm{mol}, 30.8 \mathrm{kcal} / \mathrm{mol}$, respectively with undefined enzyme classes (D-fuconate node). Hence, some empty space still remains towards discovery of novel enzymes in Methylglyoxal, Lactaldehyde and D-fuconate nodes.

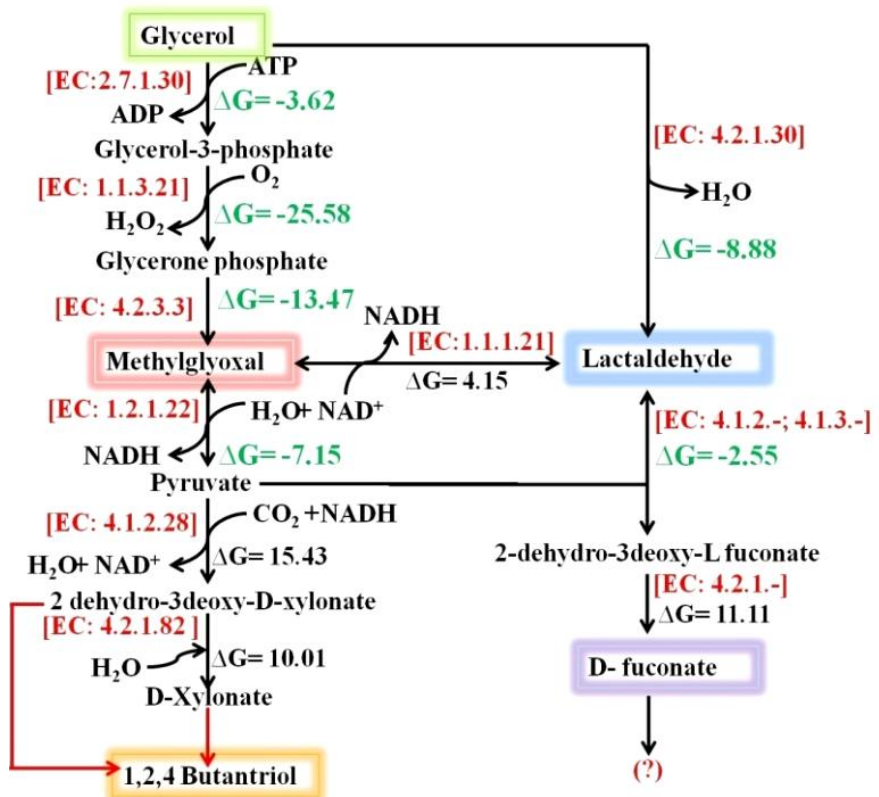

Fig 4: Predicted and designed putative synthetic metabolic network concerning glycerol entry nodes for BTO biosynthesis including thermodynamic constraints and enzyme classes (" $\Delta G$ " free energy change value represented in $\mathrm{Kcal} / \mathrm{mol}$ unit).

\section{Pitfalls of D-Xylonate downstream node}

Finally, D-Xylonate is converted into BTO following pre-existing biocatalytic reactions in downstream metabolic networks. In downstream pre-existing metabolic networks also suffer from different bottlenecks at present. The first and foremost issues are intermediate aldehyde toxicity due to untunable 2-keto acid decarboxylase $(\mathrm{MdlC})$, and thiokinase $(\mathrm{PgSucD})$ enzyme activities, imbalance in cellular redox, unfavorable physiological conditions (unavailability to redox equivalents i.e. NADH or NADPH and ATP concentration) (Sun et al., 2016), non-natural enzymatic reactions (Van Dien, 2013; Yim et al., 2011), and flux diversion due to competing pathways (Zhang et al., 2016).

\section{Solution space for BTO production}

To this end, there are plenty solution spaces still remain for further studies with careful outlook specially (a) Finding novel efficient enzymes for better catalysis and toxic intermediate clearing off, (b) Protein engineering of existing enzyme to make non-natural reactions thermodynamically-kinetically favorable, (c) Knocking out competing pathways to maximize flux towards BTO biosynthesis, (d) Implementation of micro aerobic growth condition to improve redox pool i.e. NADH, excess ATP generations for cell growth, maintenance and product transport and (e) Identification and protein engineering of specific alcohol dehydrogenases (AdhP, FucO, EutG, AdhE, YjgB, and YiaY) for prevalence of NADH over NADPH.

\section{CONCLUSION}

In general, biosynthetic pathway prediction and designing through systems biology tools are really very useful (Ghosh, 2016). It reduces time for experimentation (i.e. trial and error conventional approach for biosynthetic pathway design \& novel enzyme finding), expenses of laboratory, and manual efforts. In this study, predicted and designed synthetic metabolic pathways for D-xylonate from glycerol feedstock seems to be promising towards BTO biosynthesis, though this entire metabolic pathway needs to be validated through in vivo experimentation in suitable chassis microorganisms. These model microorganisms could be heterotrophic (Escherichia coli), and/or mixotrophic cyanobacteria (Synechococcus sp. PCC 7002, Synechococcus elongatus PCC 7942 and Synechococcus sp. PCC 6803) and green algae (Chlamydomonas reinhardtii) which can utilize crude glycerol as a carbon feedstock (towards biodiesel waste bioremediation) and sunlight as energy source (by capturing photons). Furthermore, current systems biology pathway prediction and design tools could be improved by plugging in enzyme regulations, enzyme promiscuity phenomena in near future.

\section{ACKNOWLEDGEMENTS}

I would like to thank Prof. Xin Gao, Dr. Meshari Alazmi for their immense support upon technical issues and software operations. This in silico study has been conceptualized, framed, carried out and written since March 2016 while working at ICL, London.

\section{Financial support and sponsorship: Nil.}

Conflict of Interests: There are no conflicts of interest.

\section{ABBREVIATION}

HMG-CoA: Hydroxymethylglutaryl-coenzyme A; IPP: Isopentenyl pyrophosphate; GPP: Geranyl pyrophosphate; FPP: Farnesyl pyrophosphate. PPS: Pentose phosphate shunt; NADPH: Nicotinamide adenine dinucleotide phosphate Reduced; NADH: Nicotinamide adenine dinucleotide Reduced; NADP+: Nicotinamide adenine dinucleotide phosphate oxidised; NAD+: Nicotinamide adenine dinucleotide Oxidised; EcXylA: Escherichia coli Xylose isomerase; CcXdh: Caulobacter crescentus Xylose dehydrogenase; CcXylB: Caulobacter crescentus Xylonolactonase; PfXdh: Pseudomonas fragi Xylose dehydrogenase; CcXylC: Caulobacter crescentus Xylonolactonase; EcYjhG: Escherichia coli D-Xylonate dehydratase; EcYagF: Escherichia coli D-xylonic acid dehydratase; EcYjhH: Escherichia coli 2-Keto-3-deoxy-d-xylonate Aldolase; EcYagE: Escherichia coli 2-Keto-3-deoxy-d-xylonate Aldolase; PpmdlC: Pseudomonas putida Benzoylformate 
decarboxylase; EcYiaE: Escherichia coli 2-Keto acid dehydrogenase; EcYcdW: Escherichia coli 2-Keto acid dehydrogenase; EcAdhP: Escherichia coli Aldehyde reductase; EcYqhD: Escherichia coli Alcohol dehydrogenase; ScAdh2: Saccharomyces cerevisiae Alcohol dehydrogenase 2; MtkAB: Mesorhizobium loti Malate thiokinase; PgSucD: Porphyromonas gingivalis Succinate-semialdehyde dehydrogenase; Pg4HbD: Porphyromonas gingivalis 4-Hydroxybutyrate dehydrogenase; PgAbfT-2: Porphyromonas gingivalis 4-Hydroxybutyrate CoAtransferase; EsFldA: Eggerthella sp. Cinnamoyl-CoA: phenyl lactate CoA-transferase; CbAld: Clostridium beijerinckii Coenzyme A acylating Aldehyde dehydrogenase; EcMsdh: Escherichia coli Methylmalonate-semialdehyde dehydrogenase ; EcEutG: Escherichia coli Alcohol dehydrogenase; CaAdhE2: Clostridium acetobutylicum Bifunctional aldehyde/alcohol dehydrogenase; XDG: D-xylose dehydrogenase; XDT: D-xylonate dehydratase; BFD: Benzoylformate decarboxylase ; ADG: Larabinose dehydrogenase ; ADT: L-arabinonate dehydratase ; AtDHs: Arabipsosis thaliana Dehydrogenases; AtALDHs: Arabipsosis thaliana aldehyde dehydrogenases).

\section{REFERENCES}

Abdel-Ghany SE, Day I, Heuberger AL, Broeckling CD, Reddy AS. Metabolic engineering of Arabidopsis for butanetriol production using bacterial genes. Metab Eng 2013; 20: 109-120.

Adkins H, Billica HR. The hydrogenation of esters to alcohols at 25-150 ${ }^{\circ}$. J Am Chem Soc 1948; 70: 3121-3125.

Antons S, Tilling A, Wolters E. Method for Producing Optically Active Alcohols. 2002; Patent No. US6355848.

Atkinson DE. 1977. Cellular Energy Metabolism and Its Regulation. 1st ed. New York (NY): Academic Press.

Beijer L, Nilsson RP, Holmberg C, Rutberg L. The glpP and glpF genes of the glycerol regulon in Bacillus subtilis. J Gen Microbiol 1993; 139(Pt2): 349-359.

Campbell CJ, Hinshaw JC. Making 1, 2, 4-butanetriol by hydroformylation of glycidol. 1983; United States patent No. US4410744.

Cao Y, Niu W, Guo J, Xian J, Liu H. Biotechnological production of 1,2,4-butanetriol: An efficient process to synthesize energetic material precursor from renewable biomass. Sci Rep 2015; $5: 18149$.

Casella S, Favaro L, Basaglia M. 2016. Recent Advances in Biotechnology. 1st ed. United Arab Emirates (UAE): Bentham Science Publishers.

Chou C-H, Chang W-C, Chiu C-M, Huang C-C, Huang H-D. FMM: a web server for metabolic pathway reconstruction and comparative analysis. Nucleic Acids Res. 2009; 37: W129-W134.

Dharmadi Y, Murarka A, Gonzalez R. Anaerobic fermentation of glycerol by Escherichia coli: a new platform for metabolic engineering, Biotech Bioeng 2006; 94(5): 821-829.

Flamholz A, Noor E, Bar-Even A, Ron Milo R. eQuilibrator-the biochemical thermodynamics calculator, Nucleic Acids Res. 2012; 40: D770-D775.

Frost JW, Niu W. Microbial synthesis of D-1, 2, 4-butanetriol. 2008; Patent No. WO2008/091288.

Frost JW, Niu W. Microbial synthesis of D-1, 2, 4-butanetriol. 2011; Patent No. US20110076730.

Ghosh D, Sobro IF, Hallenbeck PC. Stoichiometric conversion of biodiesel derived crude glycerol to hydrogen: Response surface methodology study of the effects of light intensity and crude glycerol and glutamate concentration. Bioresour Technol 2012a; 106: 154-160.
Ghosh D, Tourigny A, Hallenbeck PC. Near stoichiometric reforming of biodiesel derived crude glycerol to hydrogen by photofermentation, Int J Hydrogen Energy. 2012b; 37: 2273-2277.

Ghosh D. Synthetic Biology and Metabolic Engineering: a Workhorse for Novel Drugs Generations. Indo Am J Pharm Res 2016; 6(11): 7012-7025.

Gouranlou F, Kohsary I. Synthesis and characterization of 1, 2, 4-butanetrioltrinitrate. Asian J Chem 2010; 22: 4221-4228.

Ikai K, Mikami M, Furukawa Y, Ho S. Process for Producing 1, 2, 4-Butanetriol. 1999; Patent No. WO1999/044976.

Ikai K, Mikami M, Furukawa Y, Urano T, Ohtaka, S. Process for preparing 1,2,4-butanetriol. 2005; Patent No. US 6949684.

Johnson DT, Taconi KA. The glycerin glut: options for the value-added conversion of crude glycerol resulting from biodiesel production. Environ Prog 2007; 26: 338-348.

Kanehisa M, Furumichi M, Tanabe M, Sato Y, Morishima K. KEGG: new perspectives on genomes, pathways, diseases and drugs. Nucleic Acids Res 2017; 45: D353-D361.

Kerr BJ, Dozier III WA, Bregendahl K. Nutritional value of crude glycerin for nonruminants. Proceedings of the 23rd Annual Carolina Swine Nutrition Conference, Raleigh, NC 2007; pp. 6-18.

Kong W-B, Yang H, Cao Y-T, Song H, Hua S-F, Xia C-G. Effect of Glycerol and Glucose on the Enhancement of Biomass, Lipid and Soluble Carbohydrate Production by Chlorella vulgaris in Mixotrophic Culture. Food Technol. Biotechnol 2013; 51(1): 62-69.

Kuwahara H, Alazmi M, Cui X, Gao X. MRE: a web tool to suggest foreign enzymes for the biosynthesis pathway design with competing endogenous reactions in mind, Nucleic Acids Res 2016; 44:W217-W225.

Kwak BS, Kim TY, Lee SI, Kim JW. Continuous production method of 1, 2, 4-butanetriol. 2005; Patent No. WO2005/061424.

Lambert DH, Stevens SE. Photoheterotrophic growth Of Agmenellum quadruplicatum PR-6. J Bacteriol 1986; 165: 654-656.

Lau MK. Synthesis and Downstream Purification of 1, 2, 4Butanetriol. Ph.D. Thesis. 2007; Michigan State University (USA).

Li X, Cai Z, Yin Li, Zhang Y. Design and Construction of a Non-Natural Malate to 1, 2, 4-Butanetriol Pathway Creates Possibility to Produce 1, 2, 4-Butanetriol from Glucose. Sci Rep 2014; 4:5541.

Lu X, He S, Zong H, Song J. Chen W, Zhuge B. Improved 1, 2, 4-butanetriol production from an engineered Escherichia coli by coexpression of different chaperone proteins. World J Microbiol Biotechnol. 2016; 32(9):149.

Mandava VNBR, Cherukupally P, Varanasi G, Areveli S, Vempati CS, Narayana R, et al. Preparation of fosamprenavir calcium and its intermediates. 2012; Patent No. US2011/0224443A1.

May JW, Marshall JH, Sloan J. Glycerol Utilization by Schizosaccharomyces pombe: Phosphorylation of Dihydroxyacetone by a Specific Kinase as the Second Step. J Gen Microbiol 1982; 128: 1763 1766.

Molefe MN. Synthesis of D, L-1, 2, 4-butanetriol and ecaprolactam from D-flucose-derived starting materials. Ph.D. Thesis. 2005; Michigan State University (USA).

Monteith MJ, Schoefield D. Bailey, M., Process for the preparation of butanetriols. 1998; Patent No. WO1998/008793.

Murarka A, Dharmadi Y, Yazdani SS, Gonzalez R. Fermentative utilization of glycerol by Escherichia coli and its implications for the production of fuels and chemicals. Appl Environ Microbiol 2008; 74(4): 1124-1135.

Narayan MS, Manoj GP, Vatchravelu K, Bhagyalakshmi N, Mahadevaswamy M. Utilization of glycerol as carbon source on the growth, pigment and lipid production in Spirulina platensis. Int $\mathrm{J}$ Food Sci Nutr 2005; 56(7): 521-528.

Niu W, Molefe MN, Frost JW. Microbial synthesis of the energetic material precursor 1, 2, 4-butanetriol. J Am Chem Soc 2003; 125: 12998-12999.

Ren T, Liu D. Synthesis of cationic lipids from 1, 2, 4butanetriol. Tetrahedron Lett 1999; 40: 209-212. 
Ritter SK. Biomass or bust. Chem Eng News 2004; 82: 31-33.

Sun L, Yang F, Sun H, Zhu T, Li X, Li Y, et al. Synthetic pathway optimization for improved 1,2,4-butanetriol production. J Ind Microbiol Biotechnol 2016; 43: 67-78.

Swinnen S, Klein M, Carrillo M, McInnes J, Nguyen HTT, Nevoigt E. Re-evaluation of glycerol utilization in Saccharomyces cerevisiae: characterization of an isolate that grows on glycerol without supporting supplements, Biotechnol Biofuels 2013; 6:157.

Taghavi N, Robinson G. Improving the optimum yield and growth of Chlamydomonas reinhardtii $\mathrm{CC} 125$ and $\mathrm{CW} 15$ using various carbon sources and growth regimes. Afr J Biotechnol 2016; 15(23): $1083-1100$

Thompson JC, He B. Characterization of crude glycerol from biodiesel production from multiple feedstocks. Appl Eng Agric 2006; 22(2): 261-265.

Valdehuesa KNG, Liu H, Ramos KRM, Park SJ, Nisola GM, Lee W-K, et al. Direct bioconversion of d-xylose to 1,2,4-butanetriol in an engineered Escherichia coli. Process Biochem 2014; 49: 25-32.

Van Dien S. From the first drop to the first truckload: commercialization of microbial processes for renewable chemicals. Curr Opin Biotech 2013; 24: 1061-1068.

Yamada-Onodera K, Norimoto A, Kawada N, Furuya R, Yamamoto H, Tani Y, Production of Optically Active 1,2,4-Butanetriol from Corresponding Racemate by Microbial Stereo inversion. J Biosci Bioeng 2007; 103(5): 494-496.
Yamaguchi A, Hiyoshi N, Sato O, Bando KK, Shirai M. Enhancement of cyclic ether formation from polyalcohol compounds in high temperature liquid water by high pressure carbon dioxide. Green Chem 2008; 11: 48-52.

Yang F, Hanna M, Sun R. Value-added uses for crude glycerola by product of biodiesel production. Biotechnol Biofuels 2012; 5:13.

Yim H, Haselbeck R, Niu W, Pujol-Baxley C, Burgard A, Boldt $\mathrm{J}$, et al. Metabolic engineering of Escherichia coli for direct production of 1,4-butanediol. Nat Chem Biol 2011; 7: 445-452.

Zhang N, Wang J, Zhang Y, Gao H. Metabolic pathway optimization for biosynthesis of 1,2,4-butanetriol from xylose by engineered Escherichia coli. Enzyme Microb Technol 2016; 93(94): 5158 .

\section{How to cite this article:}

Ghosh D. Synthetic and Systems Biology Approach towards Designing Metabolic Bypass and Identifying Novel Enzymes for Cholesterol Lowering Drug Precursor (BTO) Biosynthesis from Crude Glycerol. J App Pharm Sci, 2017; 7 (10): 048-054. 